\title{
Récurrence de la crise frontalière : l'exception permanente en Espagne
}

Recurring Border Crises: Permanent Exception in Spain

Lorenzo Gabrielli

\section{(2) OpenEdition \\ Journals}

Édition électronique

URL : http://journals.openedition.org/conflits/19091

DOI : $10.4000 /$ conflits. 19091

ISSN : 1777-5345

Éditeur :

CECLS - Centre d'études sur les conflits - Liberté et sécurité, L'Harmattan

Édition imprimée

Date de publication : 31 décembre 2015

Pagination : 75-98

ISBN : 978-2-343-08728-3

ISSN : 1157-996X

Référence électronique

Lorenzo Gabrielli, «Récurrence de la crise frontalière : l'exception permanente en Espagne », Cultures \&

Conflits [En ligne], 99-100 | automne/hiver 2015, mis en ligne le 16 février 2017, consulté le 31 mars

2021. URL : http://journals.openedition.org/conflits/19091 ; DOI : https://doi.org/10.4000/conflits. 19091 


\section{Récurrence de la crise frontalière : I'exception permanente en Espagne *}

\section{Lorenzo GABRIELLI}

Lorenzo Gabrielli est chercheur et professeur associé an GRITIM-UPF de Barcelone et chercheur associé au Centre Émile Durkheim de Sciences Po Bordeaux. Il a travaillé sur la politique migratoire espagnole, sur l'externalisation $d u$ contrôle migratoire et ses enjeux dans les relations entre l'Europe et l'Afrique. Il a publié entre autres: "European immigration policies outside the Union. An impact analysis on migration dynamics in North African transit areas" in Jocelyne Streiff-Fénart, Aurélia Segatti (eds.), The challenge of the threshold. Border closures and migration movements in Africa, Lexington Books, Lanham MD, 2011, pp. 3-16.

T e matin du 6 février 2014, environ 300 personnes essaient de contourner à Lla nage la jetée qui sépare le littoral de Ceuta, territoire espagnol, du Maroc. Quinze d'entre elles y trouvent la mort. Les survivants témoignent que, dans un premier temps, la Guardia Civil espagnole tirait en l'air des balles en caoutchouc. Par la suite, les agents auraient commencé à viser directement les personnes dans l'eau, en particulier les parties du corps émergées, c'est-à-dire les visages et les têtes. Les témoins racontent que les agents auraient aussi utilisé du gaz lacrymogène et qu'ils auraient empêché les personnes de se rapprocher des rochers en utilisant les crosses de leurs fusils ${ }^{1}$. Les corps des 15 personnes décédées ont été enterrés à la hâte par les forces de l'ordre espagnoles, probablement pour occulter les causes des décès et leurs responsabilités. Actuellement, la justice espagnole tente d'établir les éventuelles responsabilités des agents et de leurs supérieurs.

\footnotetext{
Je tiens à remercier Flora Burchianti et Blanca Garcés Mascareñas pour leur aide et leurs commentaires très pertinents.

1. Maleno Garzón H., Sánchez Sánchez M. et Mayor Alforja I., "Informe de analisis de hechos y recopilación de testimonios de la tragedia que tuvo lugar el 6 de febrero de 2014 en la zona fronteriza de Ceuta », Caminando Fronteras, mars 2014, p. 64 [http://www.cadenaser.com/ csermedia/cadenaser/media/201403/13/espana/20140313csrcsrnac_2_Pes_PDF.pdf], page consultée le 3 mars 2015.
} 
Comment les forces de l'ordre espagnoles en sont-elles arrivées à utiliser des équipements anti-émeute face à des personnes désarmées, en claire situation de détresse, et qui ne représentaient aucune menace ? Comment un État libéral, fondé sur des principes démocratiques et le respect des droits, en est-il arrivé là, vis-à-vis d'individus qui n'étaient en train de commettre aucun délit pénal, sinon une simple infraction administrative ? Il ne s'agit là que d'un exemple parmi de nombreux autres incidents, durant ces dix dernières années. Comprendre les dynamiques qui expliquent ces agissements est d'autant plus nécessaire que les flux d'immigration vers l'Espagne sont nettement en baisse, après la sensible hausse enregistrée entre 2000 et 2008. Depuis 2011, le solde migratoire du pays est négatif ${ }^{2}$ et, aujourd'hui, l'Espagne est à nouveau considérée comme un pays d'émigration : le départ de travailleurs espagnols plus ou moins qualifiés, cherchant un emploi hors du pays, représente un phénomène toujours plus prégnant. Pourtant, la question de l'immigration irrégulière aux frontières de l'Espagne, en particulier à Ceuta et Melilla, fait régulièrement la une des quotidiens et des journaux télévisés du pays, ainsi que des autres pays européens.

Pour pouvoir comprendre les dynamiques complexes et les enjeux politiques qui ont amené à une telle gestion des flux migratoires aux frontières espagnoles, il est nécessaire de mobiliser une pluralité de concepts qui mettent en lumière les différentes dimensions de la frontière, ainsi que l'évolution de ses fonctions. En premier lieu, il est indispensable de considérer la frontière comme autre chose qu'une ligne continue et homogène. À propos de la frontière euro-méditerranéenne, X. Ferrer-Gallardo et $\mathrm{O}$. Kramsch parlent d'un «archipel frontalier », composé de différents fragments, chacun avec ses spécificités, tant du point de vue géographique et social que du point de vue des politiques vis-à-vis des flux migratoires informels ${ }^{3}$. Ces considérations peuvent être appliquées à la frontière extérieure espagnole qui est composée de plusieurs segments différents. En décomposant cette frontière, il convient de noter tout d'abord les milliers de kilomètres de la côte méditerranéenne, avec des points très "sensibles » en matière de migrations, comme la zone du détroit de Gibraltar, les localités de Tarifa, Algeciras et Cadiz, ainsi que le reste de la côte andalouse. Ensuite, il y a les espaces extra-péninsulaires, situés sur le continent africain, ou à proximité de celui-ci. Il s'agit des enclaves de Ceuta et Melilla et des 18 kilomètres de périmètre frontalier qui les entourent, ainsi que des différents îlots sous souveraineté espagnole éparpillés en face de la côte méditerranéenne marocaine. Enfin, on doit mentionner les sept îles de l'océan Atlantique qui composent l'archipel des Canaries : de l'Est à l'Ouest,

2. La Vanguardia, «El saldo migratorio español es negativo por primera vez », 16 janvier 2012 [http://www.lavanguardia.com/vida/20120116/54244485974/saldo-migratorio-espanolnegativo-primera-vez.html], page consultée le 2 mars 2015.

3. Ferrer-Gallardo X., Kramsch O. T., «El archipiélago-frente Mediterráneo : fractura, ensamblaje y movimiento en el contorno sur de la UE », in Zapata-Barrero R., Ferrer-Gallardo X. (eds.), Fronteras en movimiento. Migraciones hacia la Unión Europea en el contexto Mediterraneo, Barcelona, Edicions Bellaterra, 2012, pp. 79-102. 
Lanzarote, Fuerteventura, Grande Canarie, Tenerife, La Gomera, La Palma et El Hierro, plus des îles mineures et des îlots. Même si dans le processus de mise en scène symbolique de la frontière, celle-ci est souvent présentée par les pouvoirs publics comme un continuum, la pertinence politique de chacun de ces fragments est très différente, ainsi que les dispositifs de contrôle mis en place. C'est en considérant ces fragments différents, ainsi qu'en précisant leurs spécificités dans leur construction politique en tant que «frontière » que l'on peut déchiffrer le processus de «frontiérisation » en Espagne : il est alors possible d'identifier les diverses étapes qui le constituent et les enjeux qui le traversent.

Il est nécessaire d'analyser comment la frontière, ou plutôt les frontières espagnoles ont été construites, tant du point de vue politique et symbolique, que du point de vue des dispositifs matériels déployés. Selon W. Walters, la "frontiérisation », ainsi que la « re-frontiérisation » (re-bordering), est « une pratique sociale contingente » qui est produite «par des moyens différents, dans des contextes divers et à des fins différentes ${ }^{4}$ ». En utilisant une métaphore informatique très pertinente, cet auteur compare les frontières contemporaines à un pare-feu (firewall) qui régule les connexions entre l'intérieur et le réseau étendu à l'extérieur. Comme un firewall qui analyse et filtre les énormes flux d'informations qui entrent et sortent du système, de façon silencieuse et en arrière-plan, les frontières contemporaines filtrent les flux qui les traversent afin de « réconcilier de hauts niveaux de circulation, transmission et mouvement avec de hauts niveaux de sécurité 5 ». À cet égard, M. Kearney nous rappelle que " l'un des objectifs principaux des frontières est de servir comme filtres différentiels qui laissent passer certaines choses et pas d'autres et qui contrôlent aussi le rythme auquel passent les choses ${ }^{6}$ ». À côté de leur apparent renforcement, les frontières espagnoles apparaissent aussi comme un élément de contrôle différentiel des mobilités qui les traversent, ce qui dévoile leur " perméabilité sélective ${ }^{7}$ », caractéristique qui apparait encore plus clairement dans le cas des enclaves de Ceuta et Melilla.

Pourtant, ce fonctionnement silencieux et constant du contrôle frontalier sous forme de firewall, qui finalement est très peu visible, ne représente qu'une partie du processus de «frontiérisation » mis en place par l'État espagnol. En parallèle, il y a une autre dimension de ce processus, plus visible et évidente, qui est le centre de notre analyse ici. On se réfère à la construction

4. Walters W., "Rethinking Borders Beyond the State”, Comparative European Politics, 4/2-3, 2006, p. 145.

5. Ibid., p. 152. Toutes les traductions sont de l'auteur.

6. Kearney M., "The classifying and value-filtering missions of borders", Anthropological Theory, 4/2, 2004, p. 137.

7. Castan Pinos J., "Building Fortress Europe? Schengen and the Cases of Ceuta and Melilla”, Centre for International Border Research Working Paper, WP18, 2009 [http://www.qub.ac.uk/research-centres/CentreforInternationalBordersResearch/ Publications/WorkingPapers/CIBRWorkingPapers/], page consultée le 11 mars 2015. 
politique du caractère frontalier de certaines parties de la frontière espagnole. Ces fragments de la frontière, par leurs caractéristiques spécifiques, sont investis par le débat politique et l'attention des médias et jouent un rôle symbolique très grand dans la perception sociétale des limites territoriales et de l'action de l'État. Une duplicité des frontières existe donc, tant du point de vue des politiques qui les gèrent et des types de dispositifs de contrôle appliqués, que des caractéristiques et de l'importance quantitative des flux qui les traversent. D'un côté, il y a un contrôle en background d'une quantité énorme de flux qui traversent les frontières étatiques (échanges commerciaux, tourisme ${ }^{8}$, etc.) ; de l'autre, se produit un déploiement toujours plus visible de moyens pour faire face à un passage de personnes numériquement assez réduit mais symboliquement très impactant : les migrants irréguliers. Ces deux volets, « les invocations spectaculaires d'une crise imminente appelant à des mesures exceptionnelles » et les « routines bureaucratiques discrètes », constituent les politiques migratoires contemporaines ${ }^{9}$. De fait, ces « formes bureaucratiques et quotidiennes de contrôle », développées au fil du temps et qui se concrétisent dans des activités de data screening basées sur une "suspicion systématique $10 »$ sont occultés par la nature spectaculaire de la labellisation en crise ${ }^{11}$.

Dans le processus espagnol de «frontiérisation », les «crises migratoires », c'est-à-dire les situations labellisées d'urgence, liées à l'arrivée de migrants, jouent un rôle fondamental. Ceci est un élément marquant de la politique migratoire espagnole, où « l'urgence migratoire » concentre l'attention sur certains fragments de la frontière, qui deviennent emblématiques de cette politique, alors qu'elle vise un phénomène qui, en réalité, n’est pas forcément territorialisé et se développe plutôt ailleurs. Pour comprendre les enjeux de cette ambivalence de la politique de frontière espagnole, il faut analyser la face la plus visible de la «frontiérisation » qui concerne la composante « autonome 12 » des mouvements de personnes : celle-ci tente de subvertir la relation de pouvoir implicite dans la frontière. C’est précisément face à l'action de cette composante autonome des flux migratoires que les « crises migratoires »

8. En 2014, presque 65 millions de touristes étrangers se sont rendus en Espagne, selon les chiffres signalés par le quotidien El País; voir El País, «Epaña bate otro récord y roza los 65 millones de turistas extranjeros », 22 janvier 2015 [http://economia.elpais.com/ economia/2015/01/22/actualidad/1421928911_569617.html/].

9. Jeandesboz J., Pallister-Wilkins P., "Crisis, enforcement and control at the EU borders", in Lindley A. (ed.), Crisis and Migration. Critical perspectives, Oxford, Routledge, 2015, p. 116.

10. Jeandesboz J., "EU border control. Violence, capture and apparatus”, in Jansen J., Celikates R., de Bloois J. (eds.), The Irregularization of Migration in Contemporary Europe. Detention, deportation, drowning, London/New York, Rowman \& Littlefield, 2014, pp. 87-88.

11. Jeandesboz J., Pallister-Wilkins P., op. cit., p. 117.

12. Voir Mezzadra S., “The gaze of autonomy. Capitalism, migration and social struggles”, in Squire V. (ed.), The Contested Politics of Mobility Borderzones and Irregularity, London, Routledge, 2011, pp. 121-142. Sur l'autonomie des flux migratoires africains vers l'Espagne, voir Gabrielli L., "European Immigration Policies Outside the Union. An Impact Analysis on Migration Dynamics in North African Transit Areas”, in Streiff-Fénart J., Segatti A. (eds), The Challenge of the Threshold. Border Closures and Migration Movements in Africa, Lanham MD, Lexington Books, 2011, pp. 3-16. 
se produisent. Comme l'explique P. Cuttitta, « l'urgence est un caractère essentiel de l'actuel régime migratoire, un élément essentiel du spectacle de la frontière 13 ". Ce spectacle est " un ensemble de langages et d'images, de rhétorique, de textes et sous-textes, d'accusations et d'insinuations, ainsi qu'une grammatique visuelle qui maintient et renforce le caractère iconique d'une figure particulière et fétichisée de l'“immigration illégale” 14 ». Les pratiques matérielles de vigilance aux frontières et de contrôle de l'immigration «sont enchevêtrées dans un tissu dense de discours et de représentations, et génèrent une redondance constante de toujours plus de langages et d'images 15 ».

De façon analogue à ce qui se produit en Italie, où l'état d'urgence déclaré en 2002 a été prorogé sans cesse depuis cette date, en Espagne le gouvernement a défini à plusieurs reprises, et pour la dernière fois en mars 2014, l'arrivée de migrants irréguliers comme une «question d'État ». Il s'agit d'une déclaration politique à valeur plutôt symbolique, qui élève la question migratoire au rang de priorité de l'action gouvernementale et qui sert aussi à labelliser ces événements comme des « crises 16 ». Cette dynamique n'est pas nouvelle en Espagne et se produit aussi à un niveau moins institutionnalisé. L'utilisation par les acteurs gouvernementaux d'un lexique sensationnaliste, définissant les différents épisodes d'entrées d'étrangers aux frontières par des métaphores comme "avalanche » ou " assaut », souvent accompagnées par l'adjectif «massif », est habituelle et est aussi répandue dans la plupart des médias. Parfois, la construction de l'urgence migratoire mobilise d'autre outils, telle la relation entre immigration irrégulière et terrorisme, comme cela a été le cas récemment, lors d'une réunion en octobre 2014 à Paris, entre le ministre de l'Intérieur espagnol, Jorge Fernández Díaz, et le Commissaire européen aux Migrations et Affaires intérieures, Dimitris Avramopoulos : «il est évident - dit le ministre - que dans ces avalanches d'immigrants irréguliers se glissent aussi des terroristes djihadistes, disons le nous clairement ${ }^{17}$ ».

Le «spectacle de la frontière » bâtit « la scène d'une exclusion ostensible, dans laquelle la naturalité prétendue et la nécessité supposée de l'exclusion peuvent être démontrées et vérifiées, validées et légitimées, de manière redondante 18 ». En reprenant les considérations de M. Edelman sur la fonction symbolique des activités politiques et sur le « spectacle politique 19 », on peut

13. Cuttitta P., Lo spettacolo del confine. Lampedusa tra produzione e messa in scena della frontiera, Milano, Mimesis, 2012, p. 20.

14. De Genova N., "Spectacles of migrant 'illegality'. The scene of exclusion, the obscene of inclusion”, Ethnic and Racial Studies, 36/7, 2013, p. 1181.

15. Idem.

16. Dans le texte, nous emploierons le terme « crise » ou « crise migratoire » entre guillemets pour marquer sa labellisation politique.

17. El Mundo, «Fernández Díaz avisa de que entre los inmigrantes irregulares “se cuelan” yihadistas”, 7 novembre 2014 [http://www.elmundo.es/espana/2014/11/06/ 545ba99dca4741204c8b456d.html], page conslutée le 11 septembre 2015.

18. De Genova N., op. cit.

19. À ce propos, voir Edelman M., The Symbolic Uses of Politics, Urbana, University of Illinois 
considérer que la constante gestion de crise dans certains segments des frontières espagnoles assume une fonction de légitimation pour le gouvernement espagnol : la crise « est un puissant symbole politique contemporain » et la labellisation d'un événement comme une crise représente « une modalité cruciale pour assurer le consensus politique 20 ». Dans ce contexte, notre hypothèse est que le processus de «frontiérisation » espagnole est principalement réactif et qu'il se construit par la labellisation récurrente de "crises migratoires » sur certains segments de la ligne frontalière. Comment un phénomène structurel comme l'immigration irrégulière aux frontières espagnoles est-il codé et politisé comme « exceptionnel » ? Quelles raisons expliquent ce traitement « exceptionnel » de la part du pouvoir politique ? Quelles conséquences en découlent?

À ce propos, le cas de Ceuta et Melilla nous permet de souligner l'existence d'une «boucle de crises » (1) ainsi que le rôle des deux enclaves comme lieux privilégiés du traitement à l'urgence des arrivées de migrants irréguliers. En outre, l'analyse du rôle des îles Canaries en tant que point névralgique dans la «frontiérisation » espagnole fait émerger le caractère éphémère de cette dynamique. Nous considérons ensuite que cette «frontiérisation par les crises » cache d'autres éléments de la politique espagnole des frontières qu'il est nécessaire de décrypter. En effet, la gestion d'urgence de crises cycliques produit de facto un état d'exception et d'excès dans les segments de la frontière ayant une forte charge symbolique (2). Enfin, il nous parait nécessaire de souligner l'ambiguité d'une gestion d'urgence constante du phénomène de l'immigration irrégulière, alors qu'il s'agit désormais d'un élément structurel. À ce propos, peut-on considérer que la gestion d'urgence d'un phénomène structurel comme la migration aux frontières espagnoles constitue un moyen d'échapper aux contraintes représentées, pour l'action étatique, par le régime des droits fondamentaux (3) ?

\section{La boucle des « crises » aux frontières espagnoles}

Pour la frontière extérieure européenne, la partie espagnole qui sépare l'Espagne du Maroc et du continent africain est sans doute l'une des plus importantes, tant pour son exposition médiatique que pour ses enjeux politiques. Cependant, la signification politique des frontières espagnoles n'a pas toujours été aussi grande. En 1985, lors de l'entrée dans l'Union européenne (UE), la question de l'Espagne comme porte d'entrée des flux migratoires vers l'Europe ne se posait pas très clairement ${ }^{21}$. C'est seulement au début des

Press, 1985 (1964) et Edelman M., Constructing the Political Spectacle, Chicago/London, University of Chicago Press, 1988.

20. Jeandesboz J., Pallister-Wilkins P., op. cit., p. 115.

21. Gabrielli L., La construction de la politique d'immigration espagnole. Ambiguités et ambivalences à travers le cas des migrations onest-africaines, Thèse de Doctorat en Science Politique, LAM-Sciences Po Bordeaux, 2011, pp. 36 et suiv. [http://tel.archivesouvertes.fr/docs/00/59/91/04/PDF/Gabrielli-ThA_se_01juin2011.pdf/] 
années 1990, lors des négociations pour l'adhésion espagnole au traité de Schengen, qu'émerge nettement la nécessité de renforcer le niveau de contrôle espagnol sur l'entrée de ressortissants de pays extérieurs. À cette époque, les gouvernements des pays primo-signataires de l'accord de Schengen n'hésitent pas à considérer les frontières espagnoles comme le «soft-underbelly " le «ventre mou ») de la frontière extérieure européenne. Le gouvernement espagnol est alors poussé à renforcer minimalement ses infrastructures de contrôle frontalier, ce qui ne représentait à l'époque ni une priorité politique intérieure, ni une tâche facilement réalisable sans dépenses considérables. Dans ce cadre s'inscrit aussi l'introduction, en 1991, de l'obligation de visa pour les ressortissants marocains, algériens et tunisiens, qui rentrent dans le pays : c'est à partir de ce moment que commence à apparaître le phénomène des pateras, ces petites embarcations en bois traversant irrégulièrement le détroit de Gibraltar chargées de migrants.

À côté de cette dynamique de la «frontiérisation » espagnole impulsée par l'Europe, une composante clairement nationale, répondant à des intérêts de politique intérieure, se développe progressivement après 1999-2000 22. La politisation de la question migratoire sur la scène politique espagnole se caractérise par une apparition soudaine de cette thématique dans les débats parlementaires, par une utilisation électorale de plus en plus importante, ainsi que par un alignement des principaux partis politiques sur la nécessité de la sécurisation des frontières face à l'immigration irrégulière.

Les étapes de la «frontiérisation » espagnole, ainsi que les pièces du dispositif de blindage des frontières espagnoles sont multiples, comme le montre la chronologie indicative des «crises migratoires » aux frontières de l'Espagne qui suit.

Une revue sommaire de cette chronologie des crises migratoires aux frontières espagnoles dans les deux dernières décennies montre clairement l'existence d'une boucle de «crises » et de situations d'urgence, en même temps qu'elle souligne le caractère structurel du phénomène. La «boucle de crises » et, en conséquence, d'urgences migratoires qui touchent tour à tour de nombreuses parties du tracé frontalier espagnol nous paraît un bon exemple de la définition de la frontière comme « champ de lutte sociale permanente 23 ». Le contrôle frontalier y est plutôt une pratique basée sur la « mise à jour » (update) et la « correction/rustine 24 » patch). Cette mise à jour constante se produit en alternance dans différents points du périmètre frontalier espagnol, en parallèle avec l'apparition des « crises migratoires ».

22. Ibid, pp. 164 et suiv.

23. Walters W., op. cit., p. 152.

24. Ibid. 


\section{Chronologie indicative des principales " crises migratoires " aux frontières espagnoles}

1991 : début de l'immigration clandestine vers l'Espagne, en parallèle avec l'introduction de l'obligation de visa pour les Marocains ; apparition du phénomène des pateras transportant des migrants entre les deux rives du détroit de Gibraltar.

1998 : arrivées de migrants à Ceuta et Melilla ; dernière phase des travaux d'installation des barrières de Ceuta, commencés en 1993 ; commencement des travaux à Melilla.

1999 - années 2000 : mise en place du SIVE (Système intégré de vigilance électronique), d'abord dans la façade septentrionale du détroit de Gibraltar, ensuite dans toute la côte de la province de Cadiz (2004), sur toute la côte andalouse (2005) et aux Canaries.

2000 (février) : arrivées d'embarcations dans la zone du détroit de Gibraltar, définie par le gouvernement comme une " avalanche », en pleine campagne électorale.

2000 : entrées de migrants à Ceuta et Melilla et renforcement des dispositifs de contrôle des clôtures.

2001 (été) : arrivées de migrants dans le détroit de Gibraltar et aux îles Canaries (principalement à Fuerteventura et Grande Canarie).

2002 (décembre) : arrivées de migrants dans la province de Cadiz et aux îles Canaries.

2003 (juillet et août) : arrivées de migrants sur la côte andalouse (différentes localités de la côte de Cadix) et aux îles Canaries.

2003 (octobre) : arrivées de migrants sur la côte de Tarifa, Motril, Grenade, Almeria et aux îles Canaries (Fuerteventura).

2004 : après l'entrée en fonction du SIVE dans la zone de Gibraltar, déplacement des routes vers la mer d'Alboran, dans les provinces d'Almeria et de Grenade (pendant l'année 2004, presque trois quarts des interceptions d'embarcations de la part de la Guardia Civil en Méditerranée se réalisent dans ces provinces).

2004 (été) : arrivées de migrants à Ceuta et Melilla.

2005 (septembre, octobre) : sauts des barrières frontalières de Ceuta et Melilla par quelques centaines de personnes et mort d'une douzaine de migrants, apparemment par des tirs d'armes à feu des forces marocaines; le renforcement des dispositifs de contrôle des clôtures se poursuit.

2006 : arrivées d'environ 35000 migrants aux îles Canaries dans des embarcations venues des côtes atlantiques du continent africain (Maroc, Sahara Occidental, Mauritanie, Sénégal, Gambie, Guinée Bissau)

[...]

2012 (29 août) : 19 migrants originaires d'Afrique subsaharienne arrivent sur l'îlot de Tierra (îles de Alhucemas).

2014 (mars) : mort de 15 personnes qui essayaient d'entrer à la nage à Ceuta.

2014 : arrivées de quelques migrants aux îles Zaffarines et dans d'autres îles et îlots (la plus récente en 2014, Perejil).

2014 (11 août) : 31 bateaux gonflables transportant 299 migrants arrivent sur la côte de Cadix dans une journée (selon certaines sources, ceci serait dû à des tensions politiques avec le Maroc).

2014 (15 octobre) : saut d'environ 200 migrants tentant de franchir les grillages frontaliers pour entrer à Melilla ; ils sont accueillis de façon brutale par la Guardia Civil espagnole et refoulés immédiatement au Maroc (I'épisode est enregistré en vidéo par une association).

2014/15 : différents sauts des barrières frontalières de migrants à Melilla, renforcement des dispositifs de contrôle des clôtures. 
Nous concentrerons l'analyse principalement sur deux cas qui nous paraissent représentatifs des dynamiques qui bâtissent la «frontiérisation » espagnole : d'un côté les cas de Ceuta et Melilla et, de l'autre, celui des îles Canaries. Les deux enclaves de Ceuta et Melilla constituent les deux seuls fragments de frontière terrestre existant entre l'Europe et l'Afrique. Ces deux villes sur le continent africain représentent aussi un lieu privilégié de la « frontiérisation par les crises » et de la logique de « mise à jour » du contrôle frontalier. Les îles Canaries, quant à elles, constituent l'exemple d'un des principaux effets non prévus qui se produisent en raison du blindage d'autres parties de la frontière espagnole. Malgré sa situation dans l'océan Atlantique, à la moitié des années 2000 l'archipel exerce une fonction de prolongement de la frontière méditerranéenne entre l'Europe et l'Afrique, à cause du jeu d'action et réaction entre les politiques de contrôle et les flux migratoires, qui détermine une réorientation des routes d'immigration. La comparaison de ces deux cas nous paraît particulièrement révélatrice, étant donné que Ceuta et Melilla constituent un scénario constant dans la boucle des urgences migratoires, alors que les îles Canaries soulignent l'élément conjoncturel de la réponse étatique. Dans le cas de l'archipel, la réponse parâit en effet guidée par une logique de court terme et temporaire, qui s'accorde avec la logique spectaculaire de la gestion d'urgence, plutôt que par une vision d'ensemble du phénomène migratoire. Il est donc nécessaire de reconstruire les étapes du processus de "frontiérisation» de ces différents fragments, ainsi que de déchiffrer leurs enjeux spécifiques dans cette dynamique.

\section{Le cas de Centa et Melilla : les lieux privilégiés de la "frontiérisation par les crises»}

La logique de la politique espagnole de frontière comme une mise à jour constante des dispositifs de contrôle trouve sa représentation la plus évidente dans le cas spécifique de Ceuta et Melilla, où les « réactualisations » des barrières frontalières et des instruments de contrôle sont ininterrompues depuis les années 1990. À cet égard, Ceuta, en particulier, mais aussi Melilla ces dernières années, peuvent être considérées comme des lieux privilégiés 25 où se mettent en place tant le processus de «frontiérisation par les crises » que la gestion d'urgence de la part de l'État espagnol. Du point de vue des dispositifs matériels, l'action étatique suit principalement deux axes : d'un côté, on augmente la hauteur et le nombre des grillages frontaliers; de l'autre, on installe des instruments de contrôle s'appuyant de plus en plus sur la technologie 26 . Les premiers renforcements des barrières frontalières de Ceuta démarrent en

25. Le Boedec G., "Le détroit de Gibraltar. Les limites d'un espace modèle de la lutte européenne contre les migrations irrégulières ", EchoGéo [en ligne], 2, septembre-novembre 2007 [http://echogeo.revues.org/1488], page consultée le 19 avril 2015.

26. Ferrer-Gallardo X., "Territorial (Dis)continuity Dynamics Between Ceuta And Morocco. Conflictual Fortification vis-à-vis Cooperative Interaction at the EU Border in Africa", Tijdschrift voor Economische en Sociale Geografie, 102/1, 2011, pp. 24-38. 
1993, parallèlement à la signature espagnole de l'accord de Schengen. Cependant, les travaux avancent très lentement pour se conclure seulement en 1999, au milieu des polémiques sur la lenteur du chantier, son coût croissant et une efficacité qui apparaît déjà limitée 27 . À ce propos, il faut souligner le lien de ces travaux et de leur visibilité avec la politisation montante de la question migratoire sur la scène politique espagnole. D’ailleurs, la morphologie géographique des enclaves complique considérablement le blindage des deux périmètres frontaliers. La frontière de Ceuta passe par sept collines et présente des pentes supérieures à $35 \%$, alors que celle de Melilla est traversée par un petit fleuve ${ }^{28}$. Cependant, l'entrée de migrants à Ceuta ne se réalise pas seulement en sautant les barrières frontalières, mais aussi par d'autres moyens, comme le passage par le réseau d'égouts, qui comprend une quarantaine de connexions reliant Ceuta au territoire marocain ${ }^{29}$. Les flux migratoires irréguliers vers les deux enclaves commencent à se mettre en place dès 1991, après l'introduction de visas pour les ressortissants marocains, croissent très lentement jusqu'en 1994 et de façon plus sensible par la suite ${ }^{30}$. Pourtant, c'est seulement pendant l'été 1998 que la question des entrées de migrants vers les enclaves de Ceuta et Melilla gagne une certaine visibilité, en raison des dernières étapes des travaux de renforcement des barrières frontalières dans la ville.

Les mises à jour des dispositifs pour entraver l'entrée informelle de personnes dans les deux enclaves se poursuivent à différents moments : en 20052006, 2013, 2014 et $2015^{31}$. Une étape fondamentale du blindage frontalier de Ceuta suit les sauts des clôtures frontalières de Ceuta, en septembre et octobre 2005, quand une douzaine de migrants perdent la vie dans des circonstances toujours obscures, probablement sous les tirs des forces marocaines. À ce moment, la mise à jour des dispositifs matériels qui doivent empêcher l'entrée est très importante. La hauteur des barrières de Ceuta est relevée de 3 à 6 mètres, des diffuseurs de gaz lacrymogènes et des capteurs thermiques sont installés, ainsi qu'une troisième barrière qui s'ajoute aux deux déjà existantes ${ }^{32}$. L'enceinte qui en résulte est une triple barrière, complétée par une structure tridimensionnelle composée par des fils d'acier croisés à motifs irréguliers et destinés à empêcher les migrants de passer les trois barrières ou de

27. À cet égard, la délégation du gouvernement reconnaît déjà avant la conclusion des travaux à Ceuta que la nouvelle barrière de Melilla est plus efficace et qu'il faudra s'inspirer de celle-ci pour améliorer celle de Ceuta par la suite. El País, « Las obras de la frontera de Ceuta durarán aún seis meses más », 9 juillet 1998.

28. Soddu P., Inmigración extra-comunitaria en Europa. El caso de Centa y Melilla, Ceuta, Archivo Central, 2002, pp. 48-49.

29. El País, «La valla que costó 5.000 millones no logra frenar la avalancha de inmigrantes en Ceuta », 5 juillet 1998.

30. Témoigne de cette évolution le calendrier d'ouvertures et d'agrandissement des différents centres pour étrangers dans les deux villes, parallèle aux augmentations des entrées, comme le souligne Soddu P., op. cit., pp. 47 et suiv.

31. Gabrielli L., "La construction...", op. cit., pp. 188 et suiv.

32. Carling J., "Migration Control and Migrant Fatalities at the Spanish-African Borders", Internacional Migration Review, 41/2, 2007, p. 324. 
désactiver les systèmes de détection ${ }^{33}$. Après la mort des 15 migrants à Ceuta en mars 2014, le gouvernement espagnol renforce aussi la jetée de ciment qui sépare les eaux et la plage marocaines de celles de Ceuta. Encore une fois, ces mises à jour des barrières frontalières doivent être mises en relation avec la grande visibilité médiatique de ces épisodes. Dans la dynamique du «spectacle migratoire ", il est essentiel que la représentation de l'action étatique se produise dans un contexte à forte visibilité médiatique.

Les réactualisations des barrières de Melilla suivent un calendrier similaire. Les dix kilomètres de barrière qui encerclent Melilla commencent à être rénovés au début de l'été 1998, par l'installation de palissades en acier de trois à quatre mètres de hauteur, ainsi que de nouveaux senseurs optiques et acoustiques, des tourelles et des caméras de surveillance supplémentaires, sur sept kilomètres ${ }^{34}$. En 2005, comme à Ceuta, la hauteur des barrières de Melilla est aussi relevée à six mètres et une troisième barrière est mise en place. En 2007, on ajoute aussi une structure composée de fils d'acier croisés entre la première et la deuxième barrière pour retarder le passage des migrants. Cependant, l'importance politique des entrées à Melilla reste relativement inférieure à celle de Ceuta jusqu'à 2014, quand les tentatives de franchissement et les pratiques gouvernementales acquièrent une plus grande visibilité politique et médiatique. En effet, de nombreux témoignages graphiques ont été produits par des organisations de la société civile et diffusés par quelques organes de presse ${ }^{35}$. La réponse principale du gouvernement espagnol à cette visibilité accrue des passages à Melilla, passe encore une fois par un renforcement des dispositifs frontaliers. Tout d'abord, il s'agit de l'installation de fils barbelés Dannert ${ }^{36}$ dans une troisième partie du périmètre frontalier, ce qui suscite de fortes critiques de la part des agents sociaux et de certains partis d'opposition, en raison des blessures que ce dispositif inflige aux migrants. Cependant, le ministre de l'Intérieur a justifié son installation en soulignant encore une fois leur fonction «dissuasive ». Face à la poursuite des sauts, le gouvernement installe des éléments mobiles dans la partie supérieure des grillages frontaliers existants, augmentant leur hauteur à plus de 7 mètres. La continuation des sauts, ainsi que l'évolution des moyens utilisés pas les migrants (notamment des crampons pour les mains et des chaussures afin de faciliter l'escalade), montrent encore une fois le caractère vain de ces mises à jour constantes du blindage frontalier. La dernière étape du blindage frontalier de Melilla tient à une nou-

33. Voir les schémas techniques [http://enigmur.hypotheses.org/category/iconographies/ dispositifs-techniques].

34. EFE, « Dos vallas de acero reforzarán la frontera de Melilla », 28 juin 1998.

35. On se réfère notamment à l'association de Melilla Pro.de.in (une partie du matériel produit est accessible ici [https://vimeo.com/user12822802]), aux photojournalistes de Borders [https://vimeo.com/borderswork], ainsi qu'aux quotidiens électroniques nationaux El Diario [http://www.eldiario.es] et Público [http://www.publico.es] qui ont assuré une couverture importante et inhabituelle aux dénonciations des pratiques des forces de sécurité espagnoles.

36. Il s'agit d'un fil barbelé qui incorpore des lames de rasoir, appelé aussi fil barbelé concertina ou fil Dannert. Ce dispositif avait été déjà installé sur la barrière extérieure de la frontière de Melilla par le gouvernement Zapatero en octobre 2005, mais finalement retiré en 2007. 
velle forme d'externalisation des contrôles et concerne l'installation d'une quatrième barrière, construite sur le sol marocain par le gouvernement de Rabat, et qui court en parallèle aux trois rangées de grillages situées en territoire espagnol. Entre cette nouvelle fortification anti-migrants, composée entièrement de fils barbelés Dannert, qui longe tout le périmètre frontalier de Melilla, et les grillages espagnols, le gouvernement de Rabat a fait aussi construire une douve d'environ quatre mètres de profondeur. De plus, entre la douve et les grillages espagnols, des postes des forces armées marocaines sont distribués tout le long de la frontière, pour permettre à ces dernières d'intervenir avant que les migrants ne s'approchent des barrières espagnoles.

Le cas du contrôle frontalier à Melilla devient, entre la deuxième moitié de 2014 et 2015, très révélateur de la politique du gouvernement espagnol aussi à d'autres niveaux. Tout d'abord, il s'agit des «devoluciones en caliente ", c'està-dire des "déportations sur-le-champ ", des expulsions immédiates, collectives et sans discrimination des personnes qui viennent tout juste d'entrer sur le territoire espagnol. Selon plusieurs ONG et organisations de la société civile, il s'agit de pratiques plutôt habituelles de la part du gouvernement espagnol dans les enclaves de Ceuta et Melilla, toujours en collaboration avec le voisin marocain. Dans certaines occasions, et de façon très étonnante, ce sont directement les forces armées marocaines qui pénètrent entre les différentes barrières espagnoles pour faire descendre les migrants et les ramener directement du côté marocain de la frontière, d'où ils sont souvent acheminés, parfois encore gravement blessés, vers plusieurs localités du sud du pays ${ }^{37}$. Les pratiques du gouvernement espagnol et les annonces de réforme de la loi migratoire pour y inclure ces expulsions automatiques et sommaires entrâ̂nent des réactions très critiques de la part de plusieurs institutions internationales, ainsi que d'organisations de la société civile et de partis d'opposition en Espagne. Vers la fin octobre 2014, la Commissaire européenne aux affaires intérieures, Cecilia Malmström, envoie une lettre critique à Jorge Fernándéz Díaz, ministre de l'Intérieur espagnol. Dans sa missive, la Commissaire se réfère à l'accident filmé le 15 octobre 2014 par l'association Pro.de.in de Melilla, et regrette que les migrants qui ont dépassé les grillages frontaliers fassent « l'objet de mauvais traitements et [soient] immédiatement dirigés à la porte de la barrière avec le Maroc 38 ». Elle considère aussi que la légalisation de ces pratiques violerait les obligations espagnoles vis-à-vis du droit européen ${ }^{39}$. Le Haut commissariat des Nations unies pour les réfugiés (HCR) s'est également montré préoccupé par le recours croissant à la violence pour dissuader l'entrée des migrants et des demandeurs d'asile aux frontières espagnoles ${ }^{40}$.

37. El Diario, « Así expulsó la Guardia Civil a inmigrantes heridos e impidió la asistencia médica de Cruz Roja », 4 avril 2014 [http://www.eldiario.es/desalambre/Guardia-Civil-devolvioinmigrantes-exhaustos_0_246025495.html], page consultée le 4 avril 2015.

38. Malmström C., Lettre au Ministre espagnol de l'Intérieur Jorge Fenrandez Díaz, Bruxelles, 24-10-2014, Ref. Ares(2014)3556954 - 27/10/2014.

39. Idem. 
La dynamique de l'urgence migratoire, ainsi que ses conséquences, apparaissent clairement à Ceuta et Melilla, en raison de leurs caractéristiques géographiques, historiques et symboliques. Même si le renforcement des barrières frontalières dans les deux enclaves est un processus qui se déroule sur une longue période, il faut souligner qu'il apparait clairement que l'impulsion gouvernementale de ces mises à jour est parallèle à la labellisation en «crises migratoires». Plus récemment, les deux enclaves apparaissent aussi comme des zones où se matérialise la réponse étatique espagnole en termes d'exception et d'excès.

\section{Les îles Canaries, un point névralgique éphémère dans la boucle des crises}

L'archipel des Canaries devient progressivement une destination des flux migratoires irréguliers en provenance du continent africain, pour apparaître finalement en 2006 comme un véritable point névralgique du dispositif frontalier espagnol. La dynamique qui explique l'essor des îles Canaries comme une pièce-clé des enjeux frontaliers vis-à-vis des flux migratoires irréguliers est liée à un déplacement des routes migratoires qui se produit progressivement, et pour différentes raisons, à partir du début des années 2000. Les Canaries apparaissent alors comme une destination des flux migratoires irréguliers au départ des côtes atlantiques marocaines. Les 95 kilomètres qui séparent la côte marocaine près de la ville de Tarfaya et l'île de Fuerteventura deviennent une route alternative viable à la suite des renforcements progressifs des contrôles dans la zone du détroit de Gibraltar et dans les enclaves de Ceuta et Melilla ${ }^{41}$, ainsi qu'en raison de la coopération espagnole avec le Maroc en matière de contrôle.

Une première rupture dans la dynamique, jusqu'alors réduite, des arrivées se produit après l'installation du SIVE autour de la zone de Gibraltar, en 2004, quand les bateaux interceptés aux Canaries augmentent de $12 \%$ à $59 \%$ du total des interceptions par rapport à l'année antérieure ${ }^{42}$. Une deuxième phase du déplacement des routes migratoires vers les îles Canaries se produit en 2005, en particulier après les événements de Ceuta et Melilla de septembre et octobre, quand la pression du gouvernement espagnol pour impliquer de façon croissante le Maroc dans les opérations de contrôle s'accentue. À la suite du durcissement marocain des dispositifs de contrôle au nord du pays, les chemins migratoires vers l'Espagne se réorientent davantage vers la côte atlantique marocaine en direction des Canaries. Une intensification ultérieure du contrôle policier marocain sur sa côte atlantique pousse alors les points de

40. HCR, «Projet de loi espagnol sur les expulsions depuis les enclaves : inquiétudes du HCR », 28 octobre 2014 [http://www.unhcr.fr/544fccd5c.html/], page consultée le 18 avril 2015.

41. Le Boedec G., op. cit., p. 4. Comme le montrent les chiffres des interceptions reportées par cet auteur, dès 2002 la route par les Canaries gagne en importance par rapport au passage «classique » du détroit de Gibraltar.

42. Carling J., op. cit., p. 326. 
départ des embarcations à se déplacer toujours plus au sud, d'abord sur les côtes du Sahara occidental, ensuite vers la Mauritanie, le Sénégal et d'autres pays d'Afrique de l'Ouest ${ }^{43}$. Ce déplacement des passages migratoires vers les îles Canaries constitue un effet non prévu du blindage frontalier espagnol qui détermine une « extension » dans l'Atlantique de la «frontiérisation » espagnole commencée dans la Méditerranée. La composante autonome des flux migratoires, qui s'adaptent aux changements du contexte migratoire, amène cette réorientation des routes maritimes du détroit de Gibraltar vers la côte atlantique africaine et les Canaries.

Les arrivées de migrants en provenance d'Afrique subsaharienne aux Canaries focalisent rapidement l'attention des médias espagnols, mais aussi européens et mondiaux, transformant ainsi les îles Canaries en un point névralgique à la mode de la frontière extérieure européenne en 2006. La réponse espagnole ne se fait pas attendre et suit deux axes principaux. D'un côté, l'action gouvernementale transforme soudain l'archipel en un point névralgique du dispositif frontalier de contrôle, par l'installation du système de détection du SIVE ; puis par la mise en place des opérations de patrouille maritime Hera, sous l'égide de l'agence Frontex, commencées en juillet 2006, dans les eaux environnantes des îles, mais aussi dans les eaux territoriales du Cap-Vert, de Mauritanie et du Sénégal. De l'autre côté, l'Espagne met en place une dynamique d'externalisation du contrôle vers les pays d'Afrique occidentale, par le biais des opérations de contrôle maritime mentionnées, mais aussi de différents accords bilatéraux visant l'implication de ces pays dans le contrôle des départs et dans l'échange d'informations «sensibles » en matière d'immigration irrégulière, de réadmission et d'aide au développement ${ }^{44}$.

Cependant, l'analyse de cette "frontiérisation » soudaine est significative aussi à d'autres points de vue. D'abord, le cas des îles Canaries permet de déconstruire quelque peu le récit tant répandu de la «forteresse Europe ». Déjà en 2003, J. Carling rapporte que, lors du déploiement du SIVE dans l'île de Fuerteventura, le service maritime de la Guardia Civil est limité par des insuffisances constantes venant des travailleurs ${ }^{45}$ : personnel en congés maladie à cause de dépressions et de maladies liées au travail, effectif réduit du personnel en fonction pendant les week-ends. D'autres observations vont dans le même sens à propos du système SIVE. Ses trois stations fixes installées à partir de 2002 sur l'île de Fuerteventura, aux Canaries, utilisent une technologie radar avancée, testée par les forces armées espagnoles dans la guerre d'Afghanistan en 2001-2002. Le système a été étendu ensuite aux îles de Lanzarote, Grande Canarie et Tenerife avec la mise en place de radars mobiles, installés sur des camions ${ }^{46}$. Cette utilisation de technologies militaires déve-

43. Gabrielli L., « La construction... », op. cit., pp. 310 et suiv.

44. Pour plus de détails sur le processus de l'externalisation du contrôle menée par l'Espagne, voir Ibid., pp. 305 et suiv.

45. Carling J., op. cit., p. 337. 
loppées pour un contexte de guerre dans un contexte civil soulève des questions fondamentales. Il n'est pas toujours avéré que ces instruments soient effectivement adaptés aux fonctions de contrôle migratoire. Selon un employé du service de sauvetage maritime, lors des arrivées des pirogues en 2006, les radars des stations mobiles du SIVE sont efficaces seulement pour capter des embarcations avec une coque de métal ${ }^{47}$. Ils ne seraient alors capables de détecter ni les bateaux en bois, ni ceux en fibre de verre ou d'autres matériaux plastiques arrivant aux Canaries. La même source témoigne qu'effectivement la majorité des appels pour le sauvetage provenaient de pêcheurs ou d'embarcations commerciales qui apercevaient accidentellement les bateaux de migrants; de personnes contactées par téléphone satellitaire par les migrants eux-mêmes; ou de renseignements des officiers de liaison espagnols déplacés sur les côtes d'Afrique de l'Ouest qui récoltaient des informations sur des départs. Cela explique aussi qu'une bonne partie des embarcations de migrants qui rejoignent les Canaries dans cette période arrivent directement aux ports ou sur la plage sans être interceptées antérieurement. Cette efficacité très réduite dans la détection d'embarcations atteste encore une fois que la réponse gouvernementale espagnole est poussée par les nécessités d'une réponse spectaculaire aux arrivées de migrants. L'utilisation croissante de technologies militaires pour des tâches de contrôle migratoire souligne une fois de plus la dynamique de transformation des opérations civiles en une sorte de conflit à basse intensité, en raison des techniques utilisées, mais aussi par le nombre des victimes provoquées.

Enfin, le blindage frontalier aux Canaries se révèle très éphémère, car, moins de dix ans après son déploiement, les ressources financières allouées aux mécanismes de contrôle sont très réduites; les radars mobiles du SIVE, déjà peu efficaces auparavant, tombent en panne et leur réparation ne paraît pas une priorité ; les centres d'accueil des migrants sont presque abandonnés. De plus, la disparition des îles Canaries de la liste des points névralgiques du contrôle migratoire aux frontières extérieures de l'Europe démontre que la «frontiérisation » de cet espace a été faite par le biais de l'urgence migratoire et que, une fois la «crise » passée, elle disparait à nouveau. Le cas des îles Canaries est donc central pour mettre en exergue les allers-retours de la «frontiérisation » espagnole, dûs à sa logique principalement réactive, ainsi que pour souligner le caractère fortement conjoncturel et transitoire des dispositifs de contrôle frontalier mis en place par l'État espagnol.

46. Ibid., pp. 325-326.

47. Entretien personnel, 21 août 2013, Santa Cruz de Tenerife (Iles Canaries). 


\section{Les enjeux cachés par la cyclicité des crises : la frontière comme zone d'ex- ception et d'excès}

La gestion d'urgence du phénomène migratoire par les gouvernements espagnols, ainsi que le caractère cyclique et constant des « crises » aux frontières espagnoles, dissimulent d'autres enjeux fondamentaux liés au processus de «frontiérisation ». La face cachée de la gestion d'urgence des « crises migratoires » est la transformation de la frontière en une zone d'exception et d' " excès 48 », qui permet la réaffirmation du pouvoir étatique, appuyée sur la violence et l'extra-légalité. Il est impossible de ne pas penser ici au travail de G. Agamben 49, qui traite de l'état d'exception comme paradigme de gouvernement.

Certains auteurs, parmi lesquels T. Basaran, considèrent que les pratiques « illibérales » dans les zones de frontière ne sont pas exceptionnelles, mais suivent un processus de normalisation 50 et sont donc intégrées dans les lois et les politiques ordinaires des États libéraux ${ }^{51}$. Cependant, qu'elle soit exceptionnelle ou consubstantielle à l'action étatique, la situation qui se produit aux frontières espagnoles semble aller plus loin que la seule exclusion de l'action de l'État des normes juridiques existantes. En effet, l'état d'exception est reconnu dans la législation espagnole (Loi Organique 4/1981, 1er juin, «État d'alerte, état d'urgence et siège »), mais le gouvernement espagnol ne demande jamais formellement son application. Dans les cas en question, nous sommes confrontés à un état d'urgence de facto, qui est en dehors même du cadre juridique de l'exception. Les frontières sont utilisées de façon stratégique pour modifier l'équilibre entre la sécurité et les libertés, ainsi que pour accroître les pouvoirs de police et exclure les personnes des droits et des procédures légales ${ }^{52}$. Le cas espagnol semble confirmer cette dynamique, tant dans les déclarations que dans les pratiques. En effet, la négation des droits vis-à-vis des étrangers qui rentrent à Ceuta et Melilla se réalise par la tentative de déplacement, tant discursif que politique, de ces espaces hors des frontières du territoire national.

Lors de la rencontre avec le Commissaire européen D. Avramopoulos, le ministre Jorge Fernández Díaz, s’exprimant sur l’action étatique aux fron-

48. Andrijasevic R., "From Exception to Excess. Detention and Deportation across the Mediterranean Space”, in De Genova N., Peuts N. (eds.), The Deportation Regime. Sovereignity, Space, and the Freedom of Movement, Durham \& London, Duke University Press, 2010, pp. 147-165.

49. Agamben G., Stato di eccezione (Homo sacer II.1), Torino, Bollati Boringhieri, 2003.

50. Johns F., "Guantanamo Bay and the annihilation of the exception", The European Journal of International Law, 16/4, 2005, pp. 615 et suiv.

51. Basaran T., "Security, Law, Borders. Spaces of Exclusion”, International Political Sociology, 2, 2008, pp. 339-354 (pp. 340 et suiv.) ; voir aussi Guild E., Groenendijk K., Carrera S. (eds.), Illiberal Liberal States. Immigration, Citizenship and Integration in the EU, Londres, Ashgate, 2009.

52. Basaran T., op. cit., p. 339. 
tières contre l'immigration irrégulière et les risques de terrorisme, évoque ouvertement la tension entre sécurité et liberté : « il y a des situations dans lesquelles il est très difficile de concilier une réponse qui soit satisfaisante pour tout le monde, parce que certains mettent l'accent sur la sécurité, d'autres sur la liberté 53 ». On se réfère également aux violences policières auxquelles sont exposés les migrants ${ }^{54}$, mais aussi au cas des déportations immédiates (devoluciones en caliente) qui vont à l'encontre de la loi migratoire espagnole : celleci prescrit explicitement l'application de la procédure juridique établie pour toute personne qui rentre de façon irrégulière dans le pays avant de procéder à son retour ou son expulsion ${ }^{55}$. Le point central à considérer, comme le note T. Basaran, est la non-coïncidence de l'extension des droits fondamentaux en comparaison avec l'extension de l'action gouvernementale et des pouvoirs de police ${ }^{56}$.

Dans ce cadre, le récit politique évoquant l'intérieur et l'extérieur des frontières nationales est très utilisé dans les discours justifiant les violences policières ou le non-respect des obligations légales vis-à-vis des droits des étrangers. À propos des événements de Ceuta mentionnés au début de cet article, le ministre Jorge Fernández Díaz explique ainsi que « la géographie cède face à un concept juridique ", étant donné qu'à Ceuta et Melilla « on ne peut pas appliquer la loi de la même manière que dans le reste de l'Espagne 57 ». Le même ministre introduit un « concept opératif » de frontière qui déplace celleci à l'intérieur du territoire étatique et le détaille ainsi : "l'État repousse la ligne de vigilance jusqu'à un scénario sûr, qui n’est pas l'eau. [...] une entrée illégale [...] est achevée seulement si l'on dépasse la ligne de vigilance établie ». Selon le ministre, les personnes essayant d'entrer à Ceuta à la nage ne sont donc pas rentrées en Espagne, «étant donné qu'ils n'ont pas atteint le contrôle de police 58 ». Lors des sauts de la barrière de Melilla en 2014, et en relation avec les déportations immédiates réalisées par les forces espagnoles, le ministre déclare également que l'espace entre les trois lignes de barrières espagnoles n'est pas du territoire espagnol (en réalité la ligne de frontière séparant l'Espagne et le Maroc est bien à l'extérieur des barrières qui entourent Melilla). «Ce que certains appellent “devolución en caliente” est un rejet à la

53. El Mundo, «Fernández Díaz... », op. cit.

54. Voir : Medicos Sin Fronteras (MSF), «Violencia, vulnerabilidad y migración. Atrapados a las puertas de Europa ", 2013 [http://www.atrapadosenmarruecos.org/doc/ informemarruecos2013_cast.pdf], page consultée le 13 septembre 2015, et Amnesty International, "The Human Cost of Fortress Europe. Human Right violations against migrants and refugees at Europe's Borders”, Amnesty International Report, 2014. [https://www.amnesty.org/en/documents/EUR05/001/2014/en/], page consultée le 13 septembre 2015.

55. À ce propos, voir le rapport produit par plusieurs juristes espagnols réputés à ce sujet : Escamilla Martínez E. et al., " "Expulsiones en caliente”. Cuando el estado actúa al margen de la Ley », 2014 [http://eprints.ucm.es/25993/], page consultée le 5 avril 2015.

56. Basaran T., op. cit., pp. 340 et suiv.

57. El Mundo, « ¿Donde empieza España ? », 14 février 2014 [http://www.elmundo.es/ espana/2014/02/13/52fcc30aca47415a318b4581.html], page consultée le 11 septembre 2015.

58. Ibid. 
frontière 59 »; le ministre ajoute aussi que « tant qu'on n'a pas traversé la totalité du périmètre frontalier [les trois lignes de barrières, ndrl], bien sûr, on n'est pas en Espagne au sens de l'application des dispositions de la Loi migratoire 60 ». De même, le ministre insiste-t-il pour doter les deux enclaves espagnoles d'un « régime juridique spécial 61 ».

Dans un protocole d'action aux frontières de Ceuta et Melilla, élaboré par le ministère en octobre 2014, on explique que « exceptionnellement, on considère que l'entrée est effective pour les personnes blessés qui ont besoin d'être transportées à un centre médical 62 », sans qu'elles aient dépassé toutes les barrières. Le protocole parle explicitement de «tentatives d'assaut massives à la frontière » et fait référence à des « attitudes violentes vis-à-vis des forces de sécurité de l'État chargées de la garde [des frontières] 63 ». Le gouvernement espagnol a approuvé, le 30 mars 2015, une nouvelle " loi de sécurité citoyenne » (LOPSC 4/2015), dans laquelle se trouve un article qui légalise ces pratiques dans les cas de Ceuta et Melilla. Même si cet article est actuellement examiné par le Tribunal constitutionnel afin que soit vérifiée sa compatibilité avec les principes constitutionnels et la législation internationale, il montre bien que l'action du gouvernement de Mariano Rajoy essaye de normaliser l'exception. Cette exception se marque aussi au plan international, en référence au principe de la souveraineté d'un État à l'intérieur de ses frontières. L'entrée des forces de sécurité marocaines entre la deuxième et la troisième barrière de Melilla, pour opérer l'enlèvement de migrants sur le territoire espagnol, a une valeur symbolique particulièrement forte, étant donné les revendications territoriales historiques du Maroc sur les deux enclaves espagnoles.

En reprenant les considérations de Rutvica Andrijasevic sur la Méditerranée ${ }^{64}$, on peut considérer que, dans la gestion de la migration aux frontières d'Espagne, l'étape de l'« exception » s'accompagne de celle de l'« excès». Ceuta et Melilla constituent une bonne représentation de cette dynamique dans laquelle à l'exception fait suite l'excès, en particulier quant à la violence vis-à-vis des migrants. Cet excès de violence à laquelle les per-

59. El Diario, «Fernández Díaz contradice las pruebas judiciales: "Si un inmigrante pisa España cumplimos la Ley” », 23 septembre 2014 [http://www.eldiario.es/desalambre/FernandezDiaz-judiciales-inmigrante-Ley_0_306219557.html], page consultée le 11 septembre 2015. Traduction de l'auteur.

60. El Mundo, «Fernández Díaz defiende legalizar las devoluciones en “caliente” : "Es mejor para todos” » 23 octobre 2014 [http://www.elmundo.es/espana/2014/10/23/ 544927e522601d7c788b458f.html], page consultée le 11 septembre 2015.

61. El Mundo, "Interior considerará consumada la entrada de un inmigrante en Ceuta y Melilla si rebasa "completamente" el "sistema perimetral de seguridad” ", 22 octobre 2014 [http://www.elmundo.es/espana/2014/10/22/544803ca22601d1c2e8b456b.html], page consultée le 11 septembre 2015.

62. Ibid.

63. Ibid.

64. Andrijasevic R., op. cit. 
sonnes qui essaient de sauter les barrages frontaliers sont exposées est bien représenté par l'installation du barbelé Dannert à la frontière de Melilla. Il l'est aussi dans l'action des forces de sécurité de l'État, surtout à Ceuta et Melilla, pour ce qui concerne les agissements violents et non proportionnels à la menace portés sur des migrants désarmés, ainsi qu'aux agissements violents des forces de sécurité marocaines vis-à-vis des migrants subsahariens.

En parlant d'exception et d'excès, nous nous référons aussi à l'exceptionnalité des actions gouvernementales vis-à-vis des obligations légales, tant internationales que nationales, à l'égard des réfugiés et des potentiels demandeurs d'asile. Aux limitations structurelles déjà existantes ${ }^{65}$, ainsi qu'à celles causées par l'externalisation du contrôle migratoire vers les pays africains, s'ajoutent de nouveaux obstacles produits par les pratiques des forces de sécurité espagnoles à la frontière. Ainsi en est-il des expulsions sommaires de migrants menées contre les personnes qui viennent juste de franchir les frontières espagnoles. À cet égard, il nous semble opportun de mobiliser le concept de «néo-refoulement » pour définir une nouvelle forme de retour forcé différent $\mathrm{du}$ « non-refoulement » : une « re-spatialisation délibérée de l'asile 66 ». L'analyse se réfère en particulier au « retour des demandeurs d'asile et autres migrants vers des pays ou régions d'origine ou de transit, avant qu'ils n'atteignent le territoire où ils pourraient effectivement demander [l'asile] 67 ». Dans ce cas, parler d'externalisation de l'asile ne nous paraît pas correct, étant donné que, dans les pays de la façade méridionale de la Méditerranée, l'asile politique est de jure ou de facto inexistant. Après plusieurs débats relatifs aux implications des pratiques espagnoles vis-à-vis de l'entrée de réfugiés, le gouvernement espagnol a ouvert un bureau d'asile à la frontière espagnole de Melilla et Ceuta 68. Selon le HCR, entre septembre 2014 et mars 2015, le nouveau bureau d'asile installé au poste frontalier de Beni Enzar à Melilla a enregistré plus de 1350 demandes d'asile, déposées en grande partie par des Syriens, avec une minorité d'Irakiens et de Palestiniens ${ }^{69}$. Pourtant, la question de l'accès des demandeurs d'asile à ces bureaux se pose, étant donné que depuis l'ouverture de ce bureau, aucun ressortissant d'Afrique subsaharienne n'y aurait eu accès. En effet, au poste de frontière marocain se produirait un filtrage au faciès par

65. Entre 2007 et 2013, l'Espagne est le pays d'Europe où la différence entre la partie du budget destinée au contrôle frontalier et celle destinée au traitement des réfugiés et des demandeurs d'asile est la plus forte : respectivement 289394768 et 9342834 euros. Voir Amnesty International, Amnesty International Report, 2014, op.cit.

66. Hyndman J., Mountz A., "Another Brick in the Wall ? Neo-Refoulement and the Externalization of Asylum by Australia and Europe ", Government and Opposition, 43/2, 2008, p. 250.

67. Ibid.

68. El Diario, «Fernández Díaz inaugura las salas de asilo fronterizas antes de legalizar las expulsiones inmediatas », 17 mars 2015 [http://www.eldiario.es/desalambre/Guardia-Civildevolvio-inmigrantes-exhaustos_0_246025495.html], consulté le 4 avril 2015.

69. La Repubblica, "Melilla, apre un ufficio per i richiedenti asilo ma di fatto solo per i siriani ", 21 mars 2015 [http://www.repubblica.it/solidarieta/immigrazione/2015/03/21/news/ melilla-110152786/]. 
les militaires marocains qui rejettent les migrants noirs africains. Cette ouverture, en plus d'être un palliatif à cette situation, souligne une fois de plus le phénomène de néo-refoulement et le rôle des pays tiers voisins dans la limitation du droit d'asile.

\section{La gestion d'urgence d'un élément structurel : une échappatoire aux contraintes du régime des droits fondamentaux.}

La définition du mot «crise » renvoie à un «brusque accès » et à une «forte manifestation ». La crise est alors une rupture du statu quo, un point crucial et décisif, un moment culminant. Cependant, si l'on considère la gestion frontalière par les gouvernements espagnols, il apparaît que l'arrivée de migrants est toujours abordée comme une situation d'urgence imprévue. Après plus de vingt ans de crises et d'urgences, il est assez paradoxal que les responsables politiques présentent encore l'arrivée de quelques centaines, ou même de quelques milliers, de migrants comme une situation imprévisible constituant une «crise migratoire ». Peut-on dire qu'un phénomène est encore imprévu et inattendu lorsqu'il se répète régulièrement pendant plus de vingt ans?

La persistance du phénomène dans les deux dernières décennies met clairement en évidence le caractère structurel du phénomène. Puisqu'il s'agit d'un phénomène structurel, comment expliquer ce traitement à l'urgence permanent ? En premier lieu, nous considérons que la « frontiérisation » espagnole représente un instrument de réaffirmation symbolique et de réaffirmation de la légitimité étatique dans la gestion des « crises migratoires 70 ». En suivant $P$. Cuttitta ${ }^{71}$, on peut considérer que certains fragments de la frontière espagnole sont utilisés par les différents gouvernements espagnols comme des scénarios à forte connotation symbolique où se produit une représentation théâtralisée. Les exemples de cette fonction symbolique de la «frontiérisation » espagnole ne manquent pas. D'abord, les cas de Ceuta et Melilla, mais aussi celui des îles Canaries, où en 2006 se concentre toute l'action du gouvernement espagnol et l'attention des médias face à l'arrivé d'environ 35000 personnes. Pour souligner la disproportion entre l'importance quantitative de ces flux et leur importance symbolique et politique, il suffit de préciser que, pendant la même année, au moins 375000 immigrants sont entrés en Espagne ${ }^{72}$.

70. À ce propos voir Bigo D., «Sécurité et immigration: vers une gouvernementalité par l'inquiétude ? ", Cultures E Conflits, 31-32, 1998, pp. 13-38; voir aussi Ritaine E. (ed.), L'Europe du sud face à l'immigration. Politiques de l'Etranger, Paris, PUF, 2005.

71. Cuttitta P., op. cit., p. 20.

72. Selon le Padrón municipal, entre le 1er janvier 2006 et le 1er janvier 2007 les étrangers dans le pays et inscrits dans ce registre augmentent d'environ 375000 unités. L'année suivante, l'augmentation sera d'environ 750000 personnes. Voir INE, Estadística del Padrón continuo [http://www.ine.es/prensa/padron_tabla.htm], consulté le 5 avril 2015. 
Les cas analysés nous paraissent particulièrement significatifs aussi du fait que le blindage frontalier n'est alors pas orienté vers la construction d'une «forteresse infranchissable», mais représente la protection d'une " gated community » et défend symboliquement « une certaine manière de vivre [à l'intérieur] face à de grandes inégalités sociales 73 ». D'ailleurs, les instruments de contrôle dans les deux espaces où la gestion d'urgence est plutôt constante, Ceuta et Melilla, rappellent ceux des ces «gated communities » : la vidéosurveillance, les barrières, les patrouilles de sécurité, le dessin architectural et un examen attentif des candidats souhaitant devenir membres. Dans les deux cas, la barrière est un élément asymétrique de création de suspects : sont suspects ceux qui sont relégués à l'extérieur ${ }^{74}$.

L'action d'urgence du gouvernement espagnol vis-à-vis des crises permet aussi de déplacer la question de la sphère du débat politique à celle des questions techniques concernant les instruments du dispositif mis en place pour contrôler les frontières. Dans une dynamique de dépolitisation et de technicisation de la question du contrôle migratoire, les considérations d'ordre technique sur les solutions à appliquer deviennent le centre des discussions politiques, comme dans le cas de la question des fils barbelés pourvus de lames. Les fondements de la nécessité du contrôle ne sont alors pas remis en question et une dynamique auto-renforçante se met en place, dans laquelle la défaillance des dispositifs de sécurité des frontières est résolue avec un plus grand nombre d'appareils. Pourtant, l'arrivée ininterrompue de migrants dans certains fragments de la frontière espagnole, en plus de souligner la nature structurelle du phénomène, met également en évidence l'inefficacité des investissements continus dans le contrôle des frontières. Cela souligne de façon très évidente la faillite de la politique de frontière appliquée de façon plus ou moins enthousiaste par l'Espagne dans les dernières décennies. Il est clair que les systèmes implantés sur la frontière, et toujours remis à jour à grands frais pour les finances publiques, ne sont pas capables d'imperméabiliser la frontière face au passage de personnes. Cependant, face à de nouvelles « urgences », le pouvoir politique espagnol continue à utiliser les mêmes instruments. Le seul élément de changement tient à la quantité d'instruments appliqués, à leur modernité technologique ou à leur caractère plus dissuasif, par l'introduction d'éléments supplémentaires de menace physique.

73. Walters W., op. cit., p. 149. À propos du concept de « gated community», voir Van Houtum H., Pijpers R., "Towards a gated community", Eurozine, 6, 2003 [http://www.eurozine.com/articles/2005-01-12-houtumpijpers-en.html], consulté le 12 février 2015.

74. Ritaine E., «La barrière et le checkpoint : mise en politique de l'asymétrie », Cultures $\mathcal{E}$ Conflits, 73, 2009, p. 19. 


\section{Conclusion}

Pour revenir à l'étymologie du mot « crise », malgré son usage courant

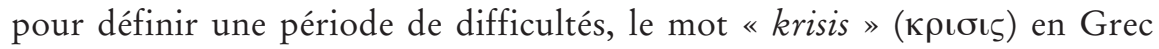
ancien signifie plutôt «faire un choix » et « décider ». La poursuite des arrivées migratoires devrait conduire plutôt à l'ouverture d'un vrai débat sur les instruments utilisés et sur une modification nécessaire de la politique migratoire et des frontières. À ce propos, le constitutionaliste J. Perez Rojo souligne que le fait que la question migratoire soit considérée comme une « question d'Etat » ne peut pas justifier l'absence d'un débat, voire l'impossibilité de critiquer l'action du gouvernement ${ }^{75}$ : selon lui, c'est justement cette importance qui doit donner lieu à un débat politique ouvert, étant donné que le gouvernement a exercé son pouvoir de façon «non légitime ». Cependant, comme le notent J. Jeandesboz et P. Pallister-Wilkins, « la crise n’implique pas seulement disruption et désordre, elle est aussi productive »; la crise permet d'utiliser « de nouveaux modes d'action ou justifie la poursuite de ceux déjà établis 76 ». Dans le cas espagnol analysé ici, les deux considérations nous paraissent, sur des plans différents, avérées. On a déjà remarqué comment la labellisation en «crise » permet au gouvernement espagnol de ne pas remettre en questions les orientations de la politique migratoire et de la gestion des frontières. Il faut aussi souligner que les « crise migratoires » et la gestion par l'urgence aux frontières espagnoles permettent au pouvoir étatique d'échapper aux contraintes imposées par le régime légal des droits, dérivant tant de la législation nationale que des obligations internationales, comme la Convention de 1951 relative au statut des réfugiés.

La conséquence est que les migrants et les réfugiés qui rentrent informellement aux frontières terrestres espagnoles se trouvent ainsi dans une zone d'exclusion des droits aux frontières, des « trous noirs juridiques ", des «zones d'exception » et de véritables «zones de pouvoir souverain pur 77 ». L'exception et l'excès aux frontières semblent donc réduire les personnes en question, qu'elles soient des migrants ou des réfugiés, à une pure «vie nue ${ }^{78}$ ».

75. El País, « Cuestión de Estado », 8 mars 2014 [http://politica.elpais.com/politica/2014/03/07/ actualidad/1394214724_764937.html], consulté le 9 avril 2015.

76. Jeandesboz J., Pallister-Wilkins P., op. cit., p. 115.

77. Basaran T., op. cit., p. 340.

78. Agamben G., Homo sacer. Il potere sovrano e la nuda vita, Torino, Einaudi, 1995. 


\section{Boîte noire méthodologique}

Mon objectif dans ce travail est de donner un cadre systématique d'interprétation aux situations d'exception et d'excès, à la violence, aux violations légales que l'on peut observer aux frontières espagnoles, en permettant d'en comprendre la dynamique, les enjeux et les éléments "structurels » qui se cachent derrière les « crises migratoires ».

II me paraît nécessaire de changer le cadre temporel de réflexion qui nous est généralement imposé par la crise et l'urgence, l'immédiateté. Changer d'échelle et considérer la " frontiérisation » espagnole sur le long terme permet de saisir les processus qui se cachent derrière cet écran de fumée représenté par les " crises migratoires ». À ce propos, les recherches que j'ai menées pendant les dernières années sur la politique d'immigration espagnole et sur son volet international en relation avec l'Afrique représentent la base sur laquelle se greffent les recherches de terrain réalisées ici.

Le choix des terrains répond à plusieurs critères. D'abord, les deux endroits choisis représentent des fragments très différents de la frontière espagnole, les enclaves de Ceuta et Melilla d'un côté et les îles Canaries de l'autre, qui ont en commun le fait d'avoir été, à des moments différents, des théâtres de crise. La focalisation de la recherche sur plusieurs localisations paraît nécessaire pour saisir les différents éléments, enjeux et stratégies mis en place par les acteurs gouvernementaux espagnols et ainsi reconstruire la dynamique plus large qui guide les politiques migratoires et les dispositifs de contrôle aux frontières. Pourtant, les caractéristiques des deux espaces de recherche analysés nous ont poussés à aborder de façon différente la recherche de terrain.

Dans le cas des îles Canaries, j'ai développé une recherche localisée pendant laquelle j'ai été surpris par l'apparente inexistence de la frontière. Tant la perception sociale, dans les îles, de la proximité avec le continent africain que les dispositifs matériels de contrôle visà-vis du phénomène migratoire paraissent avoir disparu, aussi vite qu'ils étaient apparus lors des arrivées de pirogues, au milieu des années 2000. Cela m’a obligé à reconstruire les raisons de la disparition de la perception locale d'être une frontière entre Europe et Afrique, alors qu'à distance celle-ci est considérée comme très présente. Je n'ai utilisé ici qu'une partie du matériel recueilli sur le terrain et je n'ai développé qu'une partie des réflexions menées sur ce terrain : $d$ 'autres considérations concernant le caractère colonial de la déconnexion récente entre l'archipel et l'Afrique, ainsi que les nouveaux modes de relation avec ce "voisin éloigné », seront développés ailleurs.

Dans le cas de Ceuta et Melilla, j'ai considéré que, dans un contexte d'urgence migratoire, les conditions d'enquête ne sont pas idéales pour développer une recherche cherchant à comprendre la dynamique de «frontiérisation » sur le long terme. À la transparence réduite des pratiques politiques à la frontière, en raison de la " sécuritisation " croissante de la question migratoire, s'ajoute, dans les cas de Ceuta et Melilla, la tentative du gouvernement espagnol d'occulter ses dérapages opérationnels et légaux. II est révélateur que, lors des premières phases des tentatives de saut des barrières, quelques patrouilles se préoccupent plus d'éloigner les journalistes et les témoins présents sur les lieux que les migrants, comme le rapportent plusieurs organisations de la société civile dans le cas de Melilla. À ce propos, il est crucial de mentionner le rôle de plus en plus central des organisations de la société civile, ainsi que de certains moyens de communication digitaux, pour que soient rendues visibles les pratiques du gouvernement espagnol à la frontière. La méthode appliquée pour étudier les cas de Ceuta et Melilla combine, donc, une analyse du matériel produit par les organisations de la société civile, tant espagnoles qu'internationales, avec une revue de presse basée sur des sources espagnoles imprimées et en ligne. 
Enfin, je tiens à souligner un élément méthodologique concernant le programme de recherche qui a permis de réaliser la recherche de terrain et d'écrire cet article : son caractère réellement collectif. Je voudrais relever l'importance et la richesse d'une réflexion collective profonde sur les concepts et les dynamiques étudiées pour complexifier la perception, grâce aux différentes sensibilités méthodologiques et disciplinaires. De plus, les échanges, la rétroaction des réflexions et des retours de terrains réalisés par les membres du projet permettent de complexifier la perception d'un cas isolé, et de mieux le situer dans une dynamique de "frontiérisation " plus large qui se met en place sur toute la frontière euro-méditerranéenne. 